OPEN ACCESS

Edited by:

Amy Rasley,

Lawrence Livermore National

Laboratory, USA

Reviewed by:

Olivier Dussurget,

University Paris Diderot, Institut

Pasteur, Institut National de la Santé et de la Recherche Médicale, Institut

National de la Recherche

Agronomique, France

Marisa Mariel Fernandez,

University of Buenos Aires, Instituto de

Estudios de la Inmunidad

Humoral-CONICET, Argentina

*Correspondence:

$\mathrm{Min} \mathrm{Li}$

ruth_limin@126.com;

Ying Zhang

yzhang@jhsph.edu

${ }^{\dagger}$ These authors have contributed equally to this work.

Specialty section:

This article was submitted to Microbial Immunology,

a section of the journal

Frontiers in Microbiology

Received: 12 October 2015 Accepted: 02 December 2015 Published: 23 December 2015

Citation:

Wang W, Chen J, Chen G, Du X, Cui P, Wu J, Zhao J, Wu N, Zhang W, Li M and Zhang Y (2015) Transposon

Mutagenesis Identifies Novel Genes

Associated with Staphylococcus

aureus Persister Formation.

Front. Microbiol. 6:1437.

doi: 10.3389/fmicb.2015.01437

\section{Transposon Mutagenesis Identifies Novel Genes Associated with Staphylococcus aureus Persister Formation}

\author{
Wenjie Wang ${ }^{1 \dagger}$, Jiazhen Chen ${ }^{1 \dagger}$, Gang Chen ${ }^{1}$, Xin $\mathrm{Du}^{2}$, Peng Cui ${ }^{1}$, Jing $W u^{1}$, Jing Zhao ${ }^{1}$, \\ Nan Wu ${ }^{1}$, Wenhong Zhang ${ }^{1}$, Min $\mathrm{Li}^{3 *}$ and Ying Zhang ${ }^{1,4 *}$ \\ ${ }^{1}$ Key Laboratory of Medical Molecular Virology, Huashan Hospital, Shanghai Medical College of Fudan University, Shanghai, \\ China, ${ }^{2}$ Department of Laboratory Medicine, Huashan Hospital, Shanghai Medical College of Fudan University, Shanghai, \\ China, ${ }^{3}$ Department of Laboratory Medicine, School of Medicine, Renji Hospital, Shanghai Jiao Tong University School of \\ Medicine, Shanghai, China, ${ }^{4}$ Department of Molecular Microbiology and Immunology, Johns Hopkins Bloomberg School of \\ Public Health, Johns Hopkins University, Baltimore, MD, USA
}

Pathogenic bacterial persisters are responsible for the recalcitrance of chronic and persistent infections to antimicrobial therapy. Although the mechanisms of persister formation and survival have been widely studied in Escherichia coli, persistence mechanisms in Staphylococcus aureus remain largely unknown. Here, we screened a transposon mutant library of a clinical methicillin-resistant Staphylococcus aureus(MRSA)strain, USA500 (ST8), under antibiotic pressure and identified 13 genes whose insertion mutations resulted in a defect in persistence. These candidate genes were further confirmed by evaluating the survival of the mutants upon exposure to levofloxacin and several other stress conditions. We found 13 insertion mutants with significantly lower persister numbers under several stress conditions, including sdhA, sdhB, ureG, mnhG1, fbaA, ctaB, clpX, parE, HOU_0223, HOU_0587, HOU_2091, HOU_2315, and HOU_2346, which mapped into pathways of oxidative phosphorylation, TCA cycle, glycolysis, cell cycle, and ABC transporters, suggesting that these genes and pathways may play an important role in persister formation and survival. The newly constructed knockout strains of ureG, sdhA and $s d h B$ and their complemented strains were also tested for defect in persisters following exposure to levofloxacin and several other stress conditions. The results from these experiments were consistent with the screening results, which indicated that deletion of these genes in MRSA USA500 leads to persister defect. These findings provide novel insights into the mechanisms of persister formation and survival in $S$. aureus and offer new targets for the development of persister-directed antibiotics for the improved treatment of chronic and persistent infections.

Keywords: persisters, Staphylococcus aureus, antibiotics, transposon mutant library, stress conditions

\section{INTRODUCTION}

Bacterial persisters were first described by Hobby in 1942 (Hobby et al., 1942) and have since been identified in various bacterial pathogens, such as Escherichia coli, Staphylococcus aureus, Mycobacterium tuberculosis, and Pseudomonas aeruginosa (Moyed and Bertrand, 1983; Mulcahy et al., 2010; Zhang et al., 2012). Persisters are a small bacterial population that survive a lethal 
concentration of antibiotics (Lewis, 2010). This heterogeneous subpopulation of slow- or non-growing bacterial cells regrow after removal of the antibiotic (Zhang, 2014). Studies have confirmed that persisters are the main reason for the recalcitrance of chronic and persistent infections to antimicrobial therapy, especially infections involving $S$. aureus and $P$. aeruginosa (Kint et al., 2012). S. aureus is an opportunistic pathogen that can cause severe wound and nosocomial infections. In particular, methicillin-resistant $S$. aureus (MRSA) is a major threat in hospital and community settings. Consequently, persisters pose significant challenges for the effective treatment of various infections.

The mechanisms of persister formation and survival have been studied mainly in Gram-negative bacteria, such as E. coli, and various genes and pathways have been confirmed to be involved in persister formation or survival (Zhang, 2014). The best-known pathways include toxin-antitoxin modules (HipA/B) (Moyed and Bertrand, 1983), SOS response/ DNA repair (LexA) (Debbia et al., 2001), energy production (SucB, UbiF) (Ma et al., 2010), stringent response (RelA) (Korch et al., 2003), phosphate metabolism (PhoU) (Li and Zhang, 2007), and the trans-translation mediated pathway (SsrA and SmpB) (Li et al., 2013). However, the mechanisms of persistence in Gram-positive pathogens such as $S$. aureus remain largely unknown. Stationary cultures of $S$. aureus ATCC55585 were shown to be tolerant to ciprofloxacin (Keren et al., 2004). Several genes have been confirmed to be associated with persisters in S. aureus, including hemB, mazF and $g l p K$ (Fu et al., 2009; Singh et al., 2009; Han et al., 2014). In addition, S. aureus biofilms display high tolerance to antibiotics due to the existence of persisters (Singh et al., 2009). Drug tolerance is determined by pre-existing persisters and adaptive responses after drug exposure (Lechner et al., $2012 b$ ). Due to the significant differences between Gram-positive and Gram-negative bacterial cells in terms of cell structure, gene modulation and stress response, the mechanisms of persister activity could differ between these organisms and thus warrant detailed studies.

To understand the mechanisms of persister formation and survival in $S$. aureus, we screened a transposon mutant library of the CA-MRSA clinical isolate USA500 for responses to antibiotic pressure and identified 13 genes whose mutations cause defect in persister formation.

\section{MATERIALS AND METHODS}

\section{Bacterial Strains, Plasmids, Antibiotics, and Growth Conditions}

E. coli strains DH5 $\alpha$ and TOP10 and S. aureus strain RN4220 were used in DNA cloning. The S. aureus USA500, MRSA ST8 clinical wild-type strain was used as a parent strain for screening. The plasmids pRB473 and pKOR1 were used for genetic complementation and homologous recombination, respectively (Bae and Schneewind, 2006). Antibiotics were used at the following concentrations, unless otherwise indicated: erythromycin, $5 \mu \mathrm{g} / \mathrm{ml}$; ampicillin, $100 \mu \mathrm{g} / \mathrm{ml}$; chloramphenicol, $10 \mu \mathrm{g} / \mathrm{ml}$; levofloxacin, $12.5 \mu \mathrm{g} / \mathrm{ml}$; and anhydrotetracycline
(ATc), $1 \mu \mathrm{g} / \mathrm{ml}$. Luria-Bertani (LB) broth or agar was used as the culture medium for E. coli, and tryptic soy broth (TSB) or tryptic soy agar (TSA) was used for S. aureus growth.

\section{MIC and MBC Determination}

The MIC (minimum inhibitory concentrations) and $\mathrm{MBC}$ (minimum bactericidal concentrations) of different antibiotics against the parent strain $S$. aureus USA500 and its mutants were determined as described previously (Murray, 1999). All MIC and $\mathrm{MBC}$ assays were repeated at least three times.

\section{Drug-Exposure Screen of Transposon Mutant Library Screen and Inverse PCR Identification of Mutated Genes}

The S. aureus strain USA500 was subjected to transposon mutagenesis using a previously described method ( $\mathrm{Li}$ et al., 2009). The mariner-based transposon mutagenesis exhibited no bias which was validated by inverse PCR. The transposon mutant library, which consisted of 9120 clones, was grown in TSB medium containing $5 \mu \mathrm{g} / \mathrm{ml}$ erythromycin in 96-well plates overnight at $37^{\circ} \mathrm{C}$. The library was inoculated $(1: 100)$ into fresh TSB medium using a 96-well replicator (Sigma, USA) and incubated in the 96 -well plates at $37^{\circ} \mathrm{C}$ for $16 \mathrm{~h}$ to allow the bacteria to grow to the stationary phase. Levofloxacin was added to each well at a final concentration of $12.5 \mu \mathrm{g} / \mathrm{ml}$. The drugexposed cultures were replica-transferred onto TSA plates after 3 days and 6 days of drug exposure at $37^{\circ} \mathrm{C}$.

Mutants that displayed higher susceptibility to levofloxacin relative to the parent strain after 3 or 6 days of drug exposure were selected, and the drug exposure assay was repeated to confirm the stability of the phenotype. Mutants with a decreased survival phenotype in both screening rounds were selected for identification.

Two rounds of inverse PCR were performed to locate the transposon insertions in the $S$. aureus mutants. The overnight culture of each mutant was lysed in lysis buffer (Takara, Japan) followed by boiling at $100^{\circ} \mathrm{C}$ for $10 \mathrm{~min}$. The supernatant was used as the template for the inverse PCR. The PCR primers are listed in Table S2. The first round of PCR was performed in a final volume of $25 \mu \mathrm{l}$. The first-round PCR cycling parameters were as follows: $95^{\circ} \mathrm{C}$ for $5 \mathrm{~min} ; 6$ cycles of $94^{\circ} \mathrm{C}$ for $30 \mathrm{~s}, 30^{\circ} \mathrm{C}$ for $30 \mathrm{~s}$, and $72^{\circ} \mathrm{C}$ for $1 \mathrm{~min}$; 30 cycles of $94^{\circ} \mathrm{C}$ for $30 \mathrm{~s}, 45^{\circ} \mathrm{C}$ for $30 \mathrm{~s}$, and $72^{\circ} \mathrm{C}$ for $1 \mathrm{~min}$; and $72^{\circ} \mathrm{C}$ for $5 \mathrm{~min}$. PCR $(2 \mu \mathrm{l})$ products were used as the template for the second round of PCR, which was performed at $95^{\circ} \mathrm{C}$ for $5 \mathrm{~min}$, followed by 35 cycles of $45^{\circ} \mathrm{C}$ for $30 \mathrm{~s}$ and $72^{\circ} \mathrm{C}$ for $1 \mathrm{~min}$, and finally $72^{\circ} \mathrm{C}$ for $5 \mathrm{~min}$. The PCR products of the second round were sequenced using the respective "erm" internal primers (Table S2). The insertion alleles were identified by a BLASTn search. Since USA500 was the progenitor of USA300, the USA300 genome was used as a reference.

\section{Allelic Gene Replacement and Genetic Complementation}

To knockout the candidate persistence genes $s d h A, s d h B$, and ure $G$ from the genome of USA500 wild-type, the plasmid 
pKOR1 was used to perform homologous recombination (Table $\mathrm{S} 2$ ). The allelic recombination procedure was performed as described previously (Bae and Schneewind, 2006). DNA regions located $1 \mathrm{~kb}$ upstream and downstream of the candidate genes were PCR-amplified from the chromosomal DNA of the parent strain USA500. The PCR products of the candidate genes were ligated and cloned into pKOR1 by recombination reactions (BP clonase enzyme mix, Invitrogen). The resulting plasmids were transferred to RN4220 cells and subsequently to the parent strain USA500 via electroporation. The allele replacement was performed using the following two steps: the plasmid was integrated into the chromosome by incubating the cultures at $43^{\circ} \mathrm{C}$ in TSB with $10 \mu \mathrm{g} / \mathrm{ml}$ chloramphenicol $\left(\mathrm{TSB}_{\mathrm{cm} 10}\right)$, and plasmid eviction was performed at $30^{\circ} \mathrm{C}$ in $\mathrm{TSB}_{\mathrm{cm} 10}$. The cultures were diluted $10^{4}$ times with sterile water. Then, $100 \mu \mathrm{l}$ of the diluted culture was spread on TSA containing 0,1 , or $2 \mu \mathrm{g} / \mathrm{ml}$ anhydrotetracycline (ATc) and incubated at $30^{\circ} \mathrm{C}$ for 2 days. The candidate deletion mutants were picked from TSA $\mathrm{ATc1}_{1}$ and incubated in $\mathrm{TSB}_{\mathrm{cm} 10}$ at $37^{\circ} \mathrm{C}$ overnight. Finally, chromosomal DNA was purified from the candidate mutants, which were then confirmed by PCR amplification and DNA sequencing. The primers gene-att1, gene-att2, gene-rev1, and gene-rev2 were used for allelic recombination. After the candidate genes were knocked-out, complementation strains were constructed using the shuttle vector pRB473. The candidate genes with $200 \mathrm{bp}$ upstream from the ORF which contained the predicted promoters were PCR-amplified and then cloned into pRB473. The promoters were amplified by PCR with primers Pro-F and Pro-linker, and the genes were amplified with primers genelinker and gene-R (Table S2). The recombinant plasmids were transformed into the gene deletion mutants via electroporation.

\section{Persister Assays}

To verify the defect in persistence to levofloxacin, the knockout mutants, complemented strains, and the parental strain were cultured to stationary phase $(18-20 \mathrm{~h})$ in TSB without shaking. A final concentration of $12.5 \mu \mathrm{g} / \mathrm{ml}$ levofloxacin was added to $1 \mathrm{ml}$ of the stationary-phase bacteria in 1.5-ml tubes, which were then incubated at $37^{\circ} \mathrm{C}$ without shaking. The TSA plates were used for CFU counting on days 1 to 6 by 10 -fold serial dilutions.

\section{Susceptibility to Other Stresses in Exposure Assays}

For the heat stress assay, stationary-phase cultures of the knockout mutants, complemented strains, and the parent strain were incubated at $57^{\circ} \mathrm{C}$ in a water bath for up to $3 \mathrm{~h}$; the number of CFU was then determined by plating serial dilutions of the bacteria on TSA plates.

For the oxidative stress test, stationary-phase cultures were diluted 1:100 with TSB and exposed to hydrogen peroxide $\left(\mathrm{H}_{2} \mathrm{O}_{2}\right)$ at a final concentration of $50 \mathrm{mM}$ for up to $4 \mathrm{~h}$. The survival of bacteria was determined at different times by plating serial dilutions on TSA plates.

For the acid stress test, the TSB was adjusted by adding $\mathrm{HCl}$ until the medium reached a $\mathrm{pH}$ of 3.0 and was filter-sterilized. The stationary phase cultures were harvested and washed with
TSB at $\mathrm{pH}$ 3.0. Then, the cells were resuspended in the $\mathrm{pH}-$ adjusted TSB medium and incubated at $37^{\circ} \mathrm{C}$ without shaking. The survival of the bacteria was determined after $0,1,2$, and 3 days. Control samples were subjected to the same treatment except that TSB at pH 7.0 was used. For the organic acid (acetic acid, $\mathrm{pH}$ 4.4) stress test, stationary-phase cultures were diluted 1:100 with TSB containing acetic acid at a final concentration of $80 \mathrm{mM}$. The cultures were incubated at $37^{\circ} \mathrm{C}$ without shaking for 1, 2, and 3 days. The survival of bacteria was determined by plating serial dilutions on TSA plates.

\section{Statistical Analysis}

All persister assays were repeated at least three times in this study. The data points in the graphs represent the average of three independent experiments, and error bars represent the standard deviations. A Student's $t$-test was performed for pairwise comparison, and $P$-value $<0.05$ was considered statistically significant.

\section{RESULTS}

\section{Screening For Transposon Mutants with Reduced Persister Levels}

The parent $S$. aureus strain used in this study, USA500, is a clinical isolate that is resistant to penicillin, oxacillin, cefazolin, cefuroxime, and cefoxitin and is susceptible to gentamicin, vancomycin, linezolid, levofloxacin, and rifampicin (Table S1). Because vancomycin, rifampicin, and gentamicin had very low bactericidal activity against the stationary-phase cultures, these antibiotics were not used for persister screening in this study (Figure 1). Levofloxacin had a high bactericidal activity against stationary-phase bacteria, which were decreased by 5 -log and 7log after 3- and 5-day exposure, respectively (Figure 1); therefore, levofloxacin was used to screen for mutants with reduced persister numbers. Because levofloxacin concentrations from $12.5 \mu \mathrm{g} / \mathrm{ml}(50 \times \mathrm{MIC})$ to $50 \mu \mathrm{g} / \mathrm{ml}(200 \times \mathrm{MIC})$ had almost the same killing rate (Figure S1), $12.5 \mu \mathrm{g} / \mathrm{ml}$ levofloxacin was selected for the mutant screening in this study.

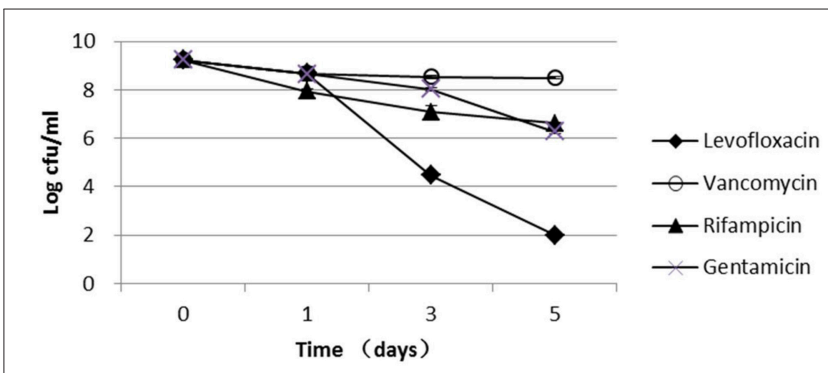

FIGURE 1 | Persister levels of the parent S. aureus strain USA500 in drug exposure assays. Stationary-phase cultures of the wild-type USA500 were exposed to the different drugs, including levofloxacin $(12.5 \mu \mathrm{g} / \mathrm{ml})$, vancomycin $(25 \mu \mathrm{g} / \mathrm{ml})$, rifampicin $(1.5 \mu \mathrm{g} / \mathrm{ml})$, and gentamicin $(100 \mu \mathrm{g} / \mathrm{ml})$. The vertical axis represents CFU values in log scale, and the horizontal axis represents the time of exposure. The error bars indicate standard deviations. 
Among the 9120 clones in the transposon mutant library, 260 were identified as having a decreased survival phenotype after 6 days of levofloxacin exposure in two rounds of screening (Figure S2). The clones exhibiting decreased survival phenotypes in the first round of screening were chosen for inclusion in the second round of screening under the same condition to ensure the accuracy of screening results. All 260 mutant clones were subjected to inverse PCR and DNA sequencing, which resulted in the identification of 13 candidate genes: $s d h A, s d h B$, ureG, mnhG1, ctaB, fbaA, clpX, parE, HOU_0223, HOU_0587, HOU_2091, HOU_2315, and HOU_2346 (Table 1). Importantly, $s d h A, s d h B, m n h G 1$, and HOU_0587 were identified from multiple clones in more than one mutant with different insertion sites, which strongly indicates their involvement in persistence. These genes belonged to pathways of oxidative phosphorylation, TCA cycle, glycolysis, cell cycle, and ABC transporters by KEGG pathway annotation.

Mutations with increased antibiotic susceptibility could also have decreased survival under antibiotic pressure. To exclude this possibility, we examined the MIC and MBC of levofloxacin for these mutants. The results showed that the MIC and MBC values of all the insertion mutants were unchanged compared with the parent strain (data not shown), suggesting that these genes are not involved in antibiotic resistance.

\section{The Candidate Mutants are More Susceptible to a Variety of Stresses}

Because genes involved in antibiotic persistence could also contribute to stress persistence (Li and Zhang, 2007; Ma et al., 2010), we tested the survival of the candidate mutants upon exposure to heat and acidic $\mathrm{pH}$ compared with the parent strain.

TABLE 1 | Candidate genes whose mutations are associated with defect in persister formation identified from the transposon mutant library of $S$. aureus USA500.

\begin{tabular}{llc}
\hline Genes & Gene products & $\begin{array}{c}\text { Number of } \\
\text { insertions }\end{array}$ \\
\hline HOU_2091 & Hypothetical membrane protein & $>5$ \\
HOU_2315 & Lysostaphin resistance protein A & $>5$ \\
sdhA & Succinate dehydrogenase flavoprotein subunit & 3 \\
sdhB & Succinate dehydrogenase iron-sulfur subunit & 2 \\
mnhG1 & Monovalent cation H+ antiporter subunit G & 2 \\
HOU_0587 & Putative pyridine nucleotide-disulfide oxidoreductase & 2 \\
ureG & Urease accessory protein UreG & 1 \\
ctaB & Protoheme IX farnesyltransferase & 1 \\
HOU_0223 & Maltose ABC transporter permease & 1 \\
fbaA & Fructose-bisphosphate aldolase & 1 \\
HOU_2346 & Response regulator of the LytR AlgR family & 1 \\
ClpX & ATP-dependent protease ATP-binding subunit ClpX & 1 \\
parE & DNA topoisomerase IV, B subunit & 1 \\
\hline HOS & & \\
\hline
\end{tabular}

All mutant clones with decreased survival phenotype were subjected to inverse PCR and DNA sequencing, and the 13 candidate genes were identified and are listed in this Table. Insertion sites indicate the number of identified insertion sites in a given gene from different mutants.
As measured in the heat $\left(57^{\circ} \mathrm{C}\right)$ and acid stress ( $\left.\mathrm{pH} 3.0\right)$ assays, ure $G, s d h A, c t a B, s d h B, c l p X, m n h G 1$, and HOU_0587 mutants were found to be more susceptible to the stresses than the parent strain (Table 2). Under acid stress, the ureG, sdhA, ctaB, $s d h B$, and HOU_0587 mutants were among the most susceptible mutants and showed a nearly 4-log decrease in CFU compared with the parent strain after 3 days of treatment. The $c l p X$, mnhG1 and HOU_0223 mutants showed 1-2 log decreases after 3 days of treatment. Similarly, under heat stress, the $u r e G, s d h B$, ctaB, clpX, and mnhG1 mutants exhibited a 1-2 log decrease in persister levels after $2 \mathrm{~h}$ of heat stress compared with the parent strain, and sdhA, parE, and HOU_2091displayed nearly 1-log decrease in persister levels. The fbaA, HOU_0223, HOU_2315, and $H O U \_2346$ mutants were not affected by either heat or acid treatment. None of the unstressed controls for the mutants or the parent strain showed decreased survival after 3 days.

\section{Decreased Persister Numbers in Newly Constructed ureG, sdhA, and sdhB Knockout Mutants in Antibiotic Exposure Assays}

To verify the role of the identified genes from the transposon mutant screening in persister formation or survival in $S$. aureus and to rule out potential polar effects of the transposon on the persister defect phenotype, we constructed fresh knockout mutants of the 3 candidate genes $u r e G, s d h A$, and $s d h B$ in $S$. aureus USA500 and their respective complements. When exposed to $12.5 \mu \mathrm{g} / \mathrm{ml}$ levofloxacin, the $\Delta u r e G, \Delta s d h A$, and $\Delta s d h B$ strains showed decreased persister levels compared with the parent strain (Figure 2). The most significant decrease occurred after 4 days of exposure to levofloxacin in all mutants. The persister levels of $u r e G, s d h A$, and $s d h B$ mutants were decreased by 1.7-log, 1.7-log and 3.5-log, respectively, relative to the parent stain after 4 days of exposure. Complementation of the $\Delta$ ure $G$ and $\Delta s d h A$ mutants with their respective wild-type genes rendered the persister levels close to that of the parent strain (Figure 2). However, complementation of $\Delta s d h B$ mutant only partially restored the the phenotype. These data indicate that ure $G$ and $s d h A$, and to a lesser extent $s d h B$, are associated with persister formation under levofloxacin treatment in $S$. aureus USA500.

\section{The Newly Constructed ureG, sdhA, and sdhB Knockout Mutants are More Susceptible to a Variety of Stresses}

Furthermore, we challenged these knockout strains with other

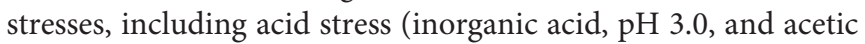
acid, $80 \mathrm{mM}, \mathrm{pH} 4.4$ ), oxidative stress (peroxide, $50 \mathrm{mM}$ ), and heat stress $\left(57^{\circ} \mathrm{C}\right)$. Since $S$. aureus has versatile responses to HCL and organic acid stress, we chose both of the inorganic acid and organic acid for stresses assays (Rode et al., 2010). For the inorganic acid stress, the persister levels of $\Delta s d h A$ and $\Delta s d h B$ mutants were 2.1-log and 2.4-log lower than the parent strain, respectively, and $\Delta u r e G$ showed a $0.7-\log$ decrease compared with the parent strain after 3 days of treatment. Complementation of these mutants restored the complemented 
TABLE 2 | Persister deficiency of the identified mutants in exposure to different stress conditions.

\begin{tabular}{|c|c|c|c|c|c|c|c|c|}
\hline \multirow[t]{3}{*}{ Genes } & \multicolumn{8}{|c|}{ No. of bacteria (Log CFU/ml, mean \pm SD) } \\
\hline & \multicolumn{4}{|c|}{ pH 3.0} & \multicolumn{4}{|c|}{$57^{\circ} \mathrm{C}$} \\
\hline & Start & $1 \mathrm{~d}$ & $2 \mathrm{~d}$ & $3 \mathbf{d}$ & $0.5 \mathrm{~h}$ & $1.0 \mathrm{~h}$ & $1.5 \mathrm{~h}$ & $2.0 \mathrm{~h}$ \\
\hline USA500 & $9.7 \pm 0.15$ & $7.8 \pm 0.17$ & $7.0 \pm 0.22$ & $4.8 \pm 0.16$ & $7.4 \pm 0.23$ & $5.7 \pm 0.24$ & $4.7 \pm 0.19$ & $4.1 \pm 0.17$ \\
\hline ureG & $9.4 \pm 0.15$ & $6.2 \pm 0.28^{\star}$ & $2.8 \pm 0.17^{\star}$ & $1.0 \pm 0.15^{\star}$ & $6.0 \pm 0.22^{\star}$ & $3.6 \pm 0.20^{\star}$ & $2.6 \pm 0.27^{\star}$ & $2.0 \pm 0.17^{\star}$ \\
\hline sdhA & $9.6 \pm 0.18$ & $6.8 \pm 0.22^{*}$ & $5.5 \pm 0.18^{\star}$ & $1.4 \pm 0.22^{*}$ & $6.9 \pm 0.27$ & $5.7 \pm 0.13$ & $4.6 \pm 0.15$ & $3.5 \pm 0.13^{*}$ \\
\hline$s d h B$ & $9.3 \pm 0.16$ & $6.3 \pm 0.23^{\star}$ & $3.7 \pm 0.22^{*}$ & $1.0 \pm 0.14^{*}$ & $6.3 \pm 0.19^{\star}$ & $4.6 \pm 0.16^{\star}$ & $3.2 \pm 0.19^{*}$ & $2.0 \pm 0.17^{*}$ \\
\hline $\operatorname{ctaB}$ & $9.4 \pm 0.27$ & $6.1 \pm 0.12^{*}$ & $2.7 \pm 0.16^{\star}$ & $1.0 \pm 0.15^{\star}$ & $5.4 \pm 0.13^{\star}$ & $4.7 \pm 0.17^{\star}$ & $4.2 \pm 0.31$ & $2.9 \pm 0.17^{*}$ \\
\hline fbaA & $9.6 \pm 0.12$ & $8.1 \pm 0.17$ & $7.5 \pm 0.16$ & $6.0 \pm 0.14$ & $6.7 \pm 0.25$ & $6.1 \pm 0.19$ & $5.2 \pm 0.13$ & $4.0 \pm 0.16$ \\
\hline clpX & $9.4 \pm 0.17$ & $7.5 \pm 0.25$ & $6.5 \pm 0.17$ & $4.8 \pm 0.17$ & $6.4 \pm 0.17$ & $5.1 \pm 0.20$ & $3.3 \pm 0.32^{*}$ & $2.1 \pm 0.27^{*}$ \\
\hline mnhG1 & $9.4 \pm 0.25$ & $7.5 \pm 0.24$ & $6.6 \pm 0.15$ & $5.1 \pm 0.19$ & $7.3 \pm 0.13$ & $5.6 \pm 0.13$ & $3.9 \pm 0.15^{\star}$ & $3.1 \pm 0.19^{*}$ \\
\hline parE & $9.6 \pm 0.17$ & $7.8 \pm 0.26$ & $6.8 \pm 0.11$ & $5.7 \pm 0.16$ & $7.1 \pm 0.16$ & $5.1 \pm 0.18$ & $3.9 \pm 0.29^{*}$ & $3.5 \pm 0.20^{*}$ \\
\hline HOU_0223 & $9.5 \pm 0.16$ & $7.5 \pm 0.21$ & $6.1 \pm 0.21$ & $4.2 \pm 0.18$ & $6.7 \pm 0.20$ & $6.3 \pm 0.13$ & $5.6 \pm 0.16$ & $3.9 \pm 0.19$ \\
\hline HOU_0587 & $9.6 \pm 0.14$ & $6.3 \pm 0.15^{\star}$ & $2.5 \pm 0.64^{\star}$ & $1.0 \pm 0.17^{\star}$ & $6.5 \pm 0.13$ & $5.3 \pm 0.20$ & $4.5 \pm 0.26$ & $4.2 \pm 0.21$ \\
\hline HOU_2091 & $9.7 \pm 0.12$ & $7.6 \pm 0.38$ & $7.3 \pm 0.17$ & $6.6 \pm 0.16$ & $7.4 \pm 0.14$ & $5.7 \pm 0.12$ & $4.1 \pm 0.12^{*}$ & $3.4 \pm 0.14^{*}$ \\
\hline HOU_2315 & $9.4 \pm 0.13$ & $7.5 \pm 0.17$ & $6.9 \pm 0.20$ & $5.8 \pm 0.18$ & $7.3 \pm 0.14$ & $5.6 \pm 0.12$ & $4.7 \pm 0.13$ & $3.9 \pm 0.21$ \\
\hline HOU_2346 & $9.5 \pm 0.16$ & $7.8 \pm 0.23$ & $6.9 \pm 0.28$ & $5.8 \pm 0.16$ & $6.6 \pm 0.27$ & $5.5 \pm 0.17$ & $4.8 \pm 0.18$ & $4.2 \pm 0.21$ \\
\hline
\end{tabular}

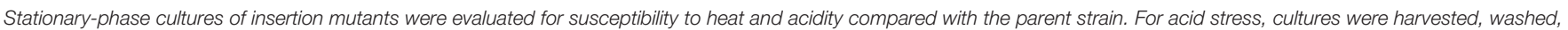

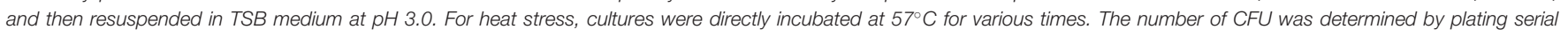
dilutions of bacteria on TSA plates.

*indicates significant differences in comparison to the parent strain (Student's $t$-test, $P$-value $<0.05$ ).

strains to the persister levels of the parent strains except $\Delta s d h B$ (Figure 3). The significant difference between $\Delta s d h B$ complementation strain and the parent strain indicated a partial complementation. The organic acetic acid stress results were different from those for the above inorganic acid, with the three knockout mutants showing only $1-\log$ decreases in persister levels compared with the parent strain USA500 after 2 days of treatment. The persister levels of the complementation strains were close to those of the parent strain except in the ureG complementation mutant (Figure 4).

In the peroxide exposure $\left(50 \mathrm{mM} \mathrm{H}_{2} \mathrm{O}_{2}\right)$ assay, the persister levels of $\Delta$ ure $G, \Delta s d h A$, and $\Delta s d h B$ mutants were 3.0-log, 2.8$\log$, and 3.4-log lower than that of the parent strain, respectively, after $3 \mathrm{~h}$ of treatment. Except for the $s d h B$ mutant, the persistence of these mutants could be partially complemented (Figure 5).

In the heat stress $\left(57^{\circ} \mathrm{C}\right)$ assay, the $\Delta u r e G, \Delta s d h A$, and $\triangle s d h B$ mutants were also more susceptible to the heat stress, showing decreased persistence by 2.2-log, 1.4-log, and 1.7$\log$, respectively, compared with the parent strain after $2 \mathrm{~h}$ of treatment. However, complementation only partially restored thepersister levels of $\Delta u r e G, \Delta s d h A$, and $\Delta s d h B$ mutants (Figure 6).

\section{DISCUSSION}

In this study, we identified 13 genes that could be associated with persister formation or survival in $S$. aureus. The inactivation by insertion mutation led to increased susceptibility to levofloxacin and other stresses. Because of the difficulty and large amount of work involved in validating all 13 genes, in this study we focused on validating the $u r e G, s d h A$, or $s d h B$ genes by constructing new knockout mutants, which were identified in multiple insertion mutants and in more than one insertion site, suggesting a strong possibility that these genes are involved in persister formation in S. aureus USA500. Another reason to construct new knockout mutants is to rule out the polar effect of transposon insertion on downstream genes. The finding that the freshly constructed ureG, $s d h A$, and $s d h B$ knockout mutants also had the same decreased persister numbers in antibiotic exposure assay as the transposon mutants demonstrated that these genes are indeed involved in persister formation. Furthermore, complementation of these genes could at least partially restore the persistence phenotypes. UreG, $s d h A$, and $s d h B$ are single genes located in a large operon. Once the operon is disrupted, gene expression may be different in genome vs. plasmid-based complementation. Additionally, the influence of other genes in the plasmid and possible compensation by other genes in the genome may also be the reasons for partially restoration. Further studies are needed to validate other insertion mutants by gene knockout in future studies.

To the best of our knowledge, this is the first comprehensive study using a transposon mutant library screen to systematically identify persister genes in $S$. aureus. Our study provides important insights into the mechanisms of persister formation in $S$. aureus and identifies new drug targets for persister-targeted antibiotic development for the improved treatment of chronic and persistent infections.

Several genes that are involved in energy production in $S$. aureus were identified in our study. The gene $f b a A$ encodes fructose-bisphosphate aldolase, which is involved in bacterial glycolysis. The $s d h A, s d h B$, and $s d h C$ genes compose the $s d h C A B$ operon and encode succinate dehydrogenase in $S$. aureus, which 


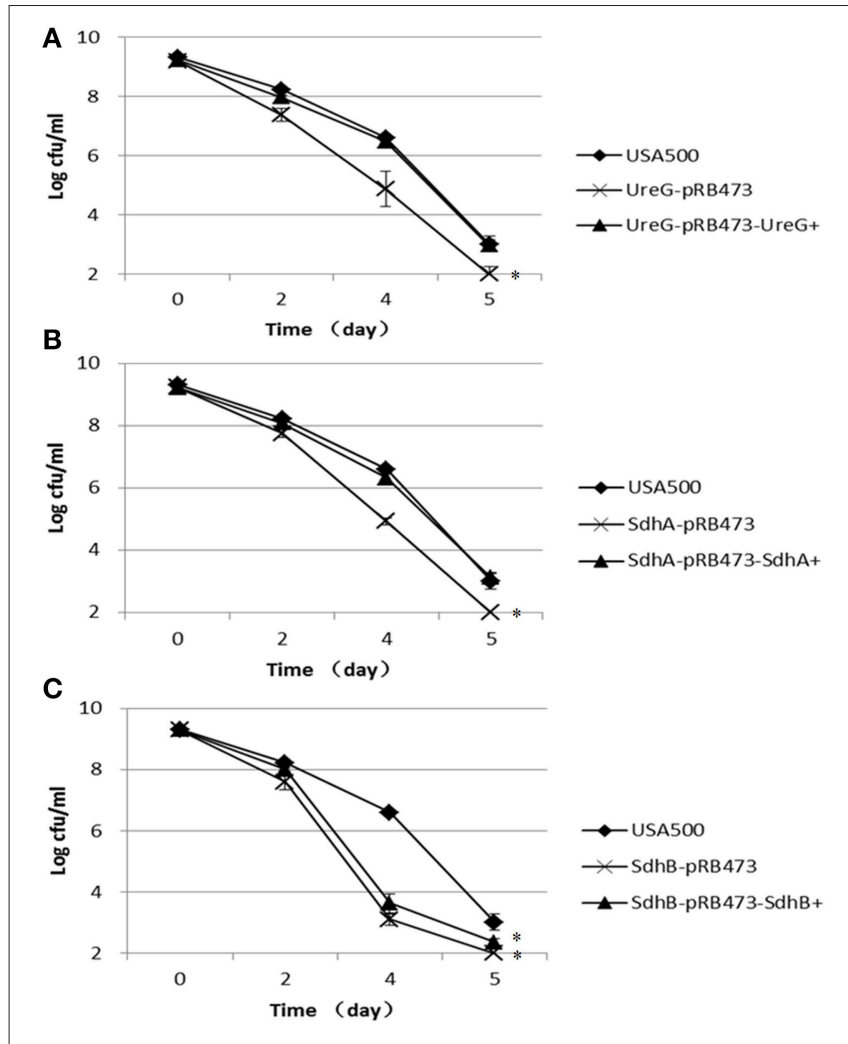

FIGURE 2 | Decreased persister levels of $\Delta u r e G, \Delta s d h A, \Delta s d h B$ mutants in levofloxacin exposure assay. Stationary-phase cultures of (A) $\Delta$ ureG, (B) $\Delta s d h A$, and (C) $\Delta s d h B$ knockout mutants, along with their complemented strains and the wild-type strain USA500, were exposed to levofloxacin $(12.5 \mu \mathrm{g} / \mathrm{ml})$. Aliquots of cultures were taken at different time points, and the dilutions were plated for CFU determination on TSA plates. The error bars indicate standard deviations. *indicates significant differences in comparison to the parent strain ( $p$-value $<0.05$ ).

is essential in the non-oxidative branch of the tricarboxylic acid (TCA) cycle. Our finding is consistent with previous studies in E. coli that showed that energy production genes, such as $s u c B$, $u b i F$, and $a c n B$, are involved in persister formation (Ma et al., 2010; Luidalepp et al., 2011). In addition, genes that are involved in the TCA cycle have been demonstrated to be upregulated in $S$. aureus biofilms; these include genes of the $s d h$ operon, 2oxoglutarate dehydrogenase $(\operatorname{odh} A)$, and succinyl-coenzyme A (CoA) synthetase (surC) (Resch et al., 2005, 2006), which may link their role in biofilms to persister formation.

Multiple clones and transposon insertion sites were identified in the $s d h A, s d h B, H O U \_0587$, and $c t a B$ genes among the persister-defective mutants in our study, which suggests that the electron transport chain may be involved in persister formation. The multiple clones that were identified in multiple insertion sites of these genes strengthen the credibility of these results and provide strong support for their role in persistence. The $s d h$ operon consists of three genes, which encode a flavoprotein that contains an FAD binding site (SdhA), an ironsulfur protein with a $4 \mathrm{Fe}-4 \mathrm{~S}$-type binding region $(\mathrm{SdhB})$ and membrane-bound cytochrome $b_{588}$ that contains two binding

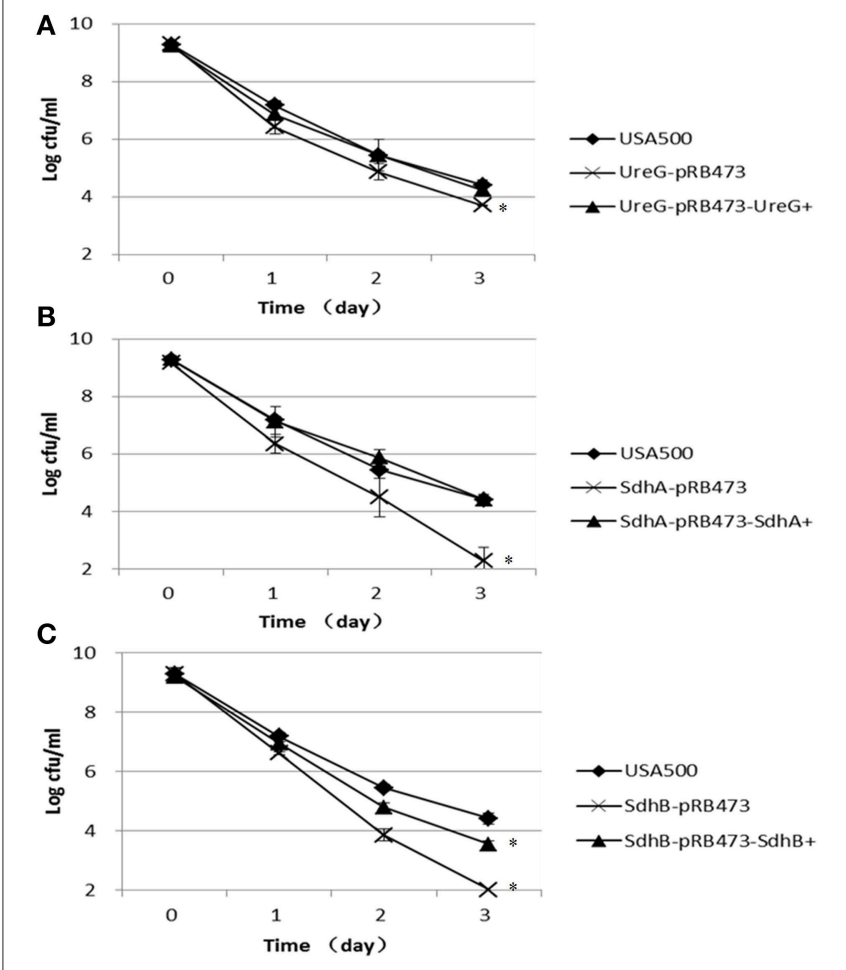

FIGURE 3 | Decreased persistence levels of $\Delta$ ureG, $\Delta s d h A, \Delta s d h B$ mutants under acid stress (pH 3.0). Stationary-phase cultures of (A) $\Delta$ ureG, (B) $\Delta s d h A$, and (C) $\Delta s d h B$ knockout mutants and their complemented strains were harvested, washed, and then resuspended in TSB medium at $\mathrm{pH}$ 3.0. The vertical axis represents CFU values in log scale, and the horizontal axis represents the time of the acid ( $\mathrm{pH} 3.0)$ treatment in days. The error bars indicate standard deviations. *indicates significant differences in comparison to the parent strain ( $p$-value $<0.05)$.

sites for heme (SdhC). The succinate dehydrogenase catalyzes the oxidation of succinate to fumarate and produces $\mathrm{FADH}_{2}$ for oxidative phosphorylation (Hederstedt and Rutberg, 1980, 1981). A previous study confirmed that ROS or free radicals produced by the electron transport chain lead to bacterial death under oxidative stress (Mols and Abee, 2011). In addition, the electron transport chain is associated with drug tolerance and virulence in S. aureus small colony variants (SCVs). SCVs have characteristic deficiencies in the electron transport chain that mainly consist of heme- and menaquinone-synthesis deficiency. Compared with wild-type levels, the persister numbers in hemB and menD mutants were significantly decreased at high concentrations of antibiotics (Lechner et al., 2012a). Mutations in other genes involved in the heme and menaquinone pathways also display SCV phenotypes, including $\operatorname{cta} A, \operatorname{men} B$, hem $A$, hem $H$, mutS, fusA, and thyA (Clements et al., 1999; Schaaff et al., 2003; Besier et al., 2007). Meanwhile, the $s d h C A B$ knockout mutant showed an SCV-like phenotype (Gaupp et al., 2010). These results indicate that the electron transport chain may play an important role in persister formation in $S$. aureus. Our findings that $s d h A, s d h B$, $H O U \_0587$, and $c t a B$ are involved in persister formation are consistent with the observation of the SCV persister phenotypes. 


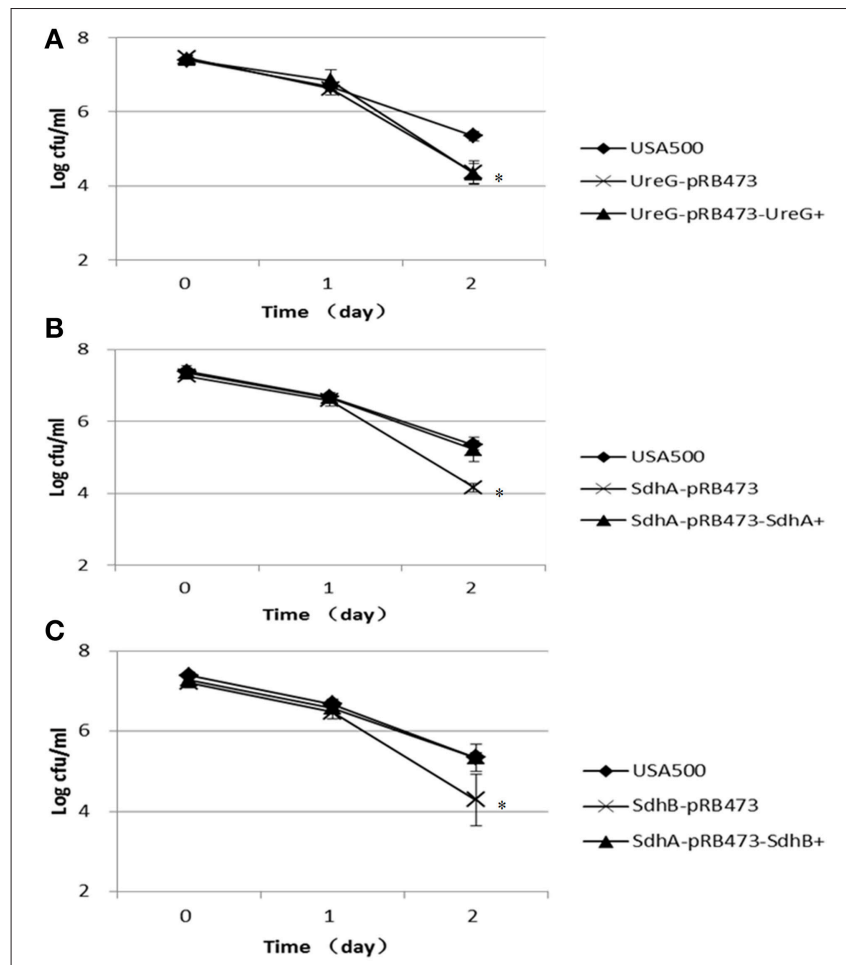

FIGURE 4 | Decreased persister levels in $\Delta u r e G, \Delta s d h A, \Delta s d h B$ mutants under organic acid stress (acetic acid). Overnight cultures were diluted 1:100 in TSB medium containing $80 \mathrm{mM}$ acetic acid. The parent strain USA500 was included as a control. The survival of the (A) $\Delta$ ureG, (B) $\Delta s d h A$, and (C) $\Delta s d h B$ knockout mutants and their complemented strains was estimated at various time points of treatment. The error bars indicate standard deviations. *indicates significant differences in comparison to the parent strain (p-value <0.05)

Future studies are needed to determine the detailed mechanisms involved.

The ureG mutant also displayed a significant decrease in persister numbers after exposure to a variety of stresses. The mechanism of persister deficiency in the ure $G$ mutant is unknown. The $u r e G$ gene encodes a subunit of urease. Ureases consist of several subunits, including structural proteins (UreA, UreB, and UreC) and accessory proteins (UreD, UreE, UreF, and UreG), and are widely present in bacteria, fungi, plants, and invertebrates. Studies have demonstrated that urease is important for bacterial survival at low $\mathrm{pH}$ in various organisms including Helicobacter pylori, Klebsiella pneumonia, and Yersinia enterocolitica (Marshall et al., 1990; Young et al., 1996; Maroncle et al., 2006). Urease is also associated with virulence in S. aureus and Brucella abortus (Highlander et al., 2007; Sangari et al., 2007). These studies indicate that the ure $G$ mutant may influence $S$. aureus virulence by affecting the activity of urease. The exact role of $u r e G$ in persister formation needs to be determined in further studies.

ClpX is a member of the Clp proteolytic complex, and its deficiency reduces biofilm formation (Frees et al., 2004). A previous study demonstrated that persisters exist in biofilmassociated S. aureus (Singh et al., 2009). Our findings indicate that

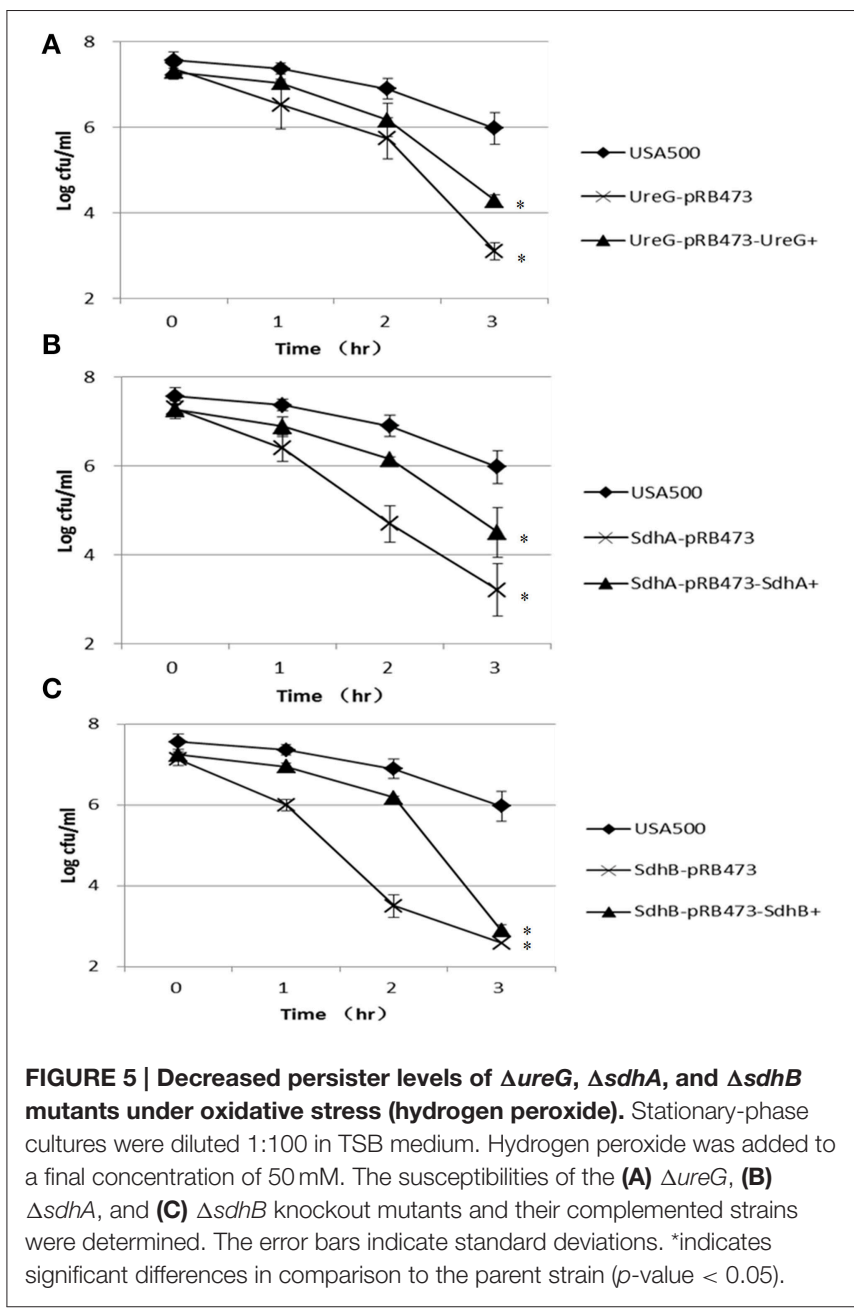

$c l p X$ plays an important role in persister and biofilm formation in $S$. aureus, although the mechanisms need to be further studied. The parE gene encodes subunit B of DNA topoisomerase IV; this enzyme is involved in DNA repair, which is known to be involved in persister formation or survival in E. coli (Debbia et al., 2001).

In addition to genes from pathways that are known to be involved in persister formation and survival, genes involved in cell transport were identified, including mnhG1 and HOU_0223. However, knockouts of these genes did not show obvious reductions in persister numbers (data not shown). The insertion transposon may partially disrupt the operon, and the partially functional genes may have different functions and therefore display different phenotypes than those associated with the wildtype genes. If the genes are deleted, the transport or sensor functions may be compensated by other genes or pathways. In this way, the reconstructed deletion mutants may lose the obvious phenotype or even acquire an opposite phenotype. For example, the phoU deletion mutant can be compensated by mutation in phoR, such that the phoU mutant does not show obvious defect in persister levels (Shi et al., unpublished), whereas the phoU transposon mutant has an obvious persister defect (Li and Zhang, 2007). 


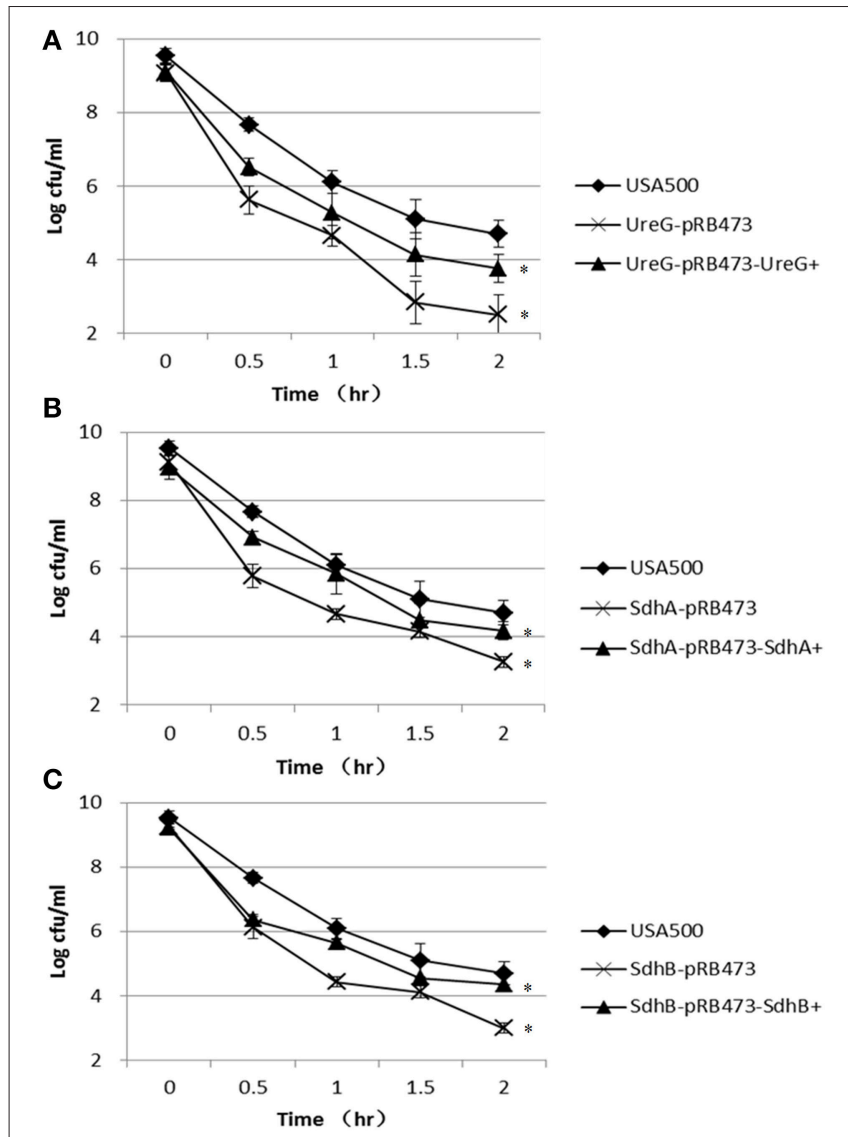

FIGURE 6 | Decreased persister levels of $\Delta u r e G, \Delta s d h A$, and $\Delta s d h B$ mutants under heat stress $\left(57^{\circ} \mathbf{C}\right)$. Stationary-phase cultures of the $(\mathbf{A})$ ureG, (B) sdhA, and (C) sdhB mutants, complemented strains and the parent strain were incubated at $57^{\circ} \mathrm{C}$ for various times, and the number of $\mathrm{CFU}$ was determined by plating serial dilutions of bacteria on TSA plates. The error bars indicate standard deviations. *indicates significant differences in comparison to the parent strain ( $p$-value $<0.05)$.

One limitation of the study is the gene coverage of the transposon mutant library. The multiple independent mutant hits for the top hits (Table 1) while reassuring in confirming the role of these genes in persister phenotype, may suggest

\section{REFERENCES}

Bae, T., and Schneewind, O. (2006). Allelic replacement in Staphylococcus aureus with inducible counter-selection. Plasmid 55, 58-63. doi: 10.1016/j.plasmid.2005.05.005

Besier, S., Ludwig, A., Ohlsen, K., Brade, V., and Wichelhaus, T. A. (2007). Molecular analysis of the thymidine-auxotrophic small colony variant phenotype of Staphylococcus aureus. Int. J. Med. Microbiol. 297, 217-225. doi: 10.1016/j.ijmm.2007.02.003

Clements, M. O., Watson, S. P., Poole, R. K., and Foster, S. J. (1999). CtaA of Staphylococcus aureus is required for starvation survival, recovery, and cytochrome biosynthesis. J. Bacteriol. 181, 501-507.

Debbia, E. A., Roveta, S., Schito, A. M., Gualco, L., and Marchese, A. (2001). Antibiotic persistence: the role of spontaneous DNA repair a potential bias in the mutant library. In fact, to avoid the transposon integration bias of the previously used transposon, we used the mariner-based plasmid for transposon mutagenesis. In our previous virulence studies with the same transposon mutant library, no apparent bias for mariner transposon integration was found ( $\mathrm{Li}$ et al., 2009). However, multiple insertions in HOU_2091 and HOU_2315 mutants indicate the possibility of a bias of the mutant library in this study (Table 1). Thus, certain genes that are important in persister formation and survival may have been missed in this study. Future studies with more clones or with different mutant libraries may be needed to more comprehensively identify $S$. aureus persister genes.

In summary, we identified 13 novel genes which may associated with persister survival and formation in S. aureus. These genes are involved in several pathways including energy production, electron transport chain, DNA repair, biofilm formation, and cell transport. These findings provide novel insights into the mechanisms of persister formation and survival in S. aureus, and offer new targets for developing new antibiotics that kill persisters for improved treatment of chronic and persistent infections.

\section{AUTHOR CONTRIBUTIONS}

$\mathrm{YZ}, \mathrm{ML}$, and WZ designed the work and revised the manuscript; WW, JC, GC, XD, and PC completed all the experiments; JW, JZ, and NW performed the statistically analysis and made the figures; WW and JC, YZ wrote the manuscript.

\section{ACKNOWLEDGMENTS}

This work was supported by the National Natural Science Foundation of China (81101226, 81471987, and 81572046) and the Program of Shanghai Subject Chief Scientist (13XD1401200). YZ was supported in part by NIH grants AI099512 and AI108535.

\section{SUPPLEMENTARY MATERIAL}

The Supplementary Material for this article can be found online at: http://journal.frontiersin.org/article/10.3389/fmicb. 2015.01437 773347

Frees, D., Chastanet, A., Qazi, S., Sørensen, K., Hill, P., Msadek, T., et al. (2004). Clp ATPases are required for stress tolerance, intracellular replication and biofilm formation in Staphylococcus aureus. Mol. Microbiol. 54, 1445-1462. doi: 10.1111/j.1365-2958.2004. 04368.x

Fu, Z., Tamber, S., Memmi, G., Donegan, N. P., and Cheung, A. L. (2009). Overexpression of MazFsa in Staphylococcus aureus induces bacteriostasis by selectively targeting mRNAs for cleavage. J. Bacteriol. 191, 2051-2059. doi: 10.1128/JB.00907-08

Gaupp, R., Schlag, S., Liebeke, M., Lalk, M., and Götz, F. (2010). Advantage of upregulation of succinate dehydrogenase in Staphylococcus aureus biofilms. J. Bacteriol. 192, 2385-2394. doi: 10.1128/JB.01472-09 
Han, J., He, L., Shi, W., Xu, X., Wang, S., Zhang, S., et al. (2014). Glycerol uptake is important for l-form formation and persistence in Staphylococcus aureus. PLoS ONE 9:e108325. doi: 10.1371/journal.pone.0108325

Hederstedt, L., and Rutberg, L. (1980). Biosynthesis and membrane binding of succinate dehydrogenase in Bacillus subtilis. J. Bacteriol. 144, 941-951.

Hederstedt, L., and Rutberg, L. (1981). Succinate dehydrogenase-a comparative review. Microbiol. Rev. 45, 542-555.

Highlander, S. K., Hulten, K. G., Qin, X., Jiang, H., Yerrapragada, S., Mason, E. O. Jr., et al. (2007). Subtle genetic changes enhance virulence of methicillin resistant and sensitive Staphylococcus aureus. BMC Microbiol. 7:99. doi: 10.1186/1471-2180-7-99

Hobby, G. L., Meyer, K., and Chaffee, E. (1942). Observations on the mechanism of action of penicillin. Proc. Soc. Exp. Biol. Med. 50, 281-285. doi: 10.3181/00379727-50-13773

Keren, I., Kaldalu, N., Spoering, A., Wang, Y., and Lewis, K. (2004). Persister cells and tolerance to antimicrobials. FEMS Microbiol. Lett. 230, 13-18. doi: 10.1016/S0378-1097(03)00856-5

Kint, C. I., Verstraeten, N., Fauvart, M., and Michiels, J. (2012). New-found fundamentals of bacterial persistence. Trends Microbiol. 20, 577-585. doi: 10.1016/j.tim.2012.08.009

Korch, S. B., Henderson, T. A., and Hill, T. M. (2003). Characterization of the hipA7 allele of Escherichia coli and evidence that high persistence is governed by (p)ppGpp synthesis. Mol. Microbiol. 50, 1199-1213. doi: 10.1046/j.13652958.2003.03779.x

Lechner, S., Lewis, K., and Bertram, R. (2012a). Staphylococcus aureus persisters tolerant to bactericidal antibiotics. J. Mol. Microbiol. Biotechnol. 22, 235-244. doi: $10.1159 / 000342449$

Lechner, S., Patra, P., Klumpp, S., and Bertram, R. (2012b). Interplay between population dynamics and drug tolerance of Staphylococcus aureus persister cells. J. Mol. Microbiol. Biotechnol. 22, 381-391. doi: 10.1159/000346073

Lewis, K. (2010). Persister cells. Annu. Rev. Microbiol. 64, 357-372. doi: 10.1146/annurev.micro.112408.134306

Li, J., Ji, L., Shi, W., Xie, J., and Zhang, Y. (2013). Trans-translation mediates tolerance to multiple antibiotics and stresses in Escherichia coli. J. Antimicrob. Chemother. 68, 2477-2481. doi: 10.1093/jac/dkt231

Li, M., Rigby, K., Lai, Y., Nair, V., Peschel, A., Schittek, B., et al. (2009). Staphylococcus aureus mutant screen reveals interaction of the human antimicrobial peptide dermcidin with membrane phospholipids. Antimicrob. Agents Chemother. 53, 4200-4210. doi: 10.1128/AAC.00428-09

Li, Y., and Zhang, Y. (2007). PhoU is a persistence switch involved in persister formation and tolerance to multiple antibiotics and stresses in Escherichia coli. Antimicrob. Agents Chemother. 51, 2092-2099. doi: 10.1128/AAC.00052-07

Luidalepp, H., Jõers, A., Kaldalu, N., and Tenson, T. (2011). Age of inoculum strongly influences persister frequency and can mask effects of mutations implicated in altered persistence. J. Bacteriol. 193, 3598-3605. doi: 10.1128/JB.00085-11

Ma, C., Sim, S., Shi, W., Du, L., Xing, D., and Zhang, Y. (2010). Energy production genes sucB and ubiF are involved in persister survival and tolerance to multiple antibiotics and stresses in Escherichia coli. FEMS Microbiol. Lett. 303, 33-40. doi: 10.1111/j.1574-6968.2009.01857.x

Maroncle, N., Rich, C., and Forestier, C. (2006). The role of Klebsiella pneumoniae urease in intestinal colonization and resistance to gastrointestinal stress. Res. Microbiol. 157, 184-193. doi: 10.1016/j.resmic.2005.06.006

Marshall, B. J., Barrett, L. J., Prakash, C., McCallum, R. W., and Guerrant, R. L. (1990). Urea protects Helicobacter (Campylobacter) pylori from the bactericidal effect of acid. Gastroenterology 99, 697-702.
Mols, M., and Abee, T. (2011). Primary and secondary oxidative stress in Bacillus. Environ. Microbiol. 13, 1387-1394. doi: 10.1111/j.1462-2920.2011. 02433.x

Moyed, H. S., and Bertrand, K. P. (1983). hipA, a newly recognized gene of Escherichia coli K-12 that affects frequency of persistence after inhibition of murein synthesis. J. Bacteriol. 155, 768-775.

Mulcahy, L. R., Burns, J. L., Lory, S., and Lewis, K. (2010). Emergence of Pseudomonas aeruginosa strains producing high levels of persister cells in patients with cystic fibrosis. J. Bacteriol. 192, 6191-6199. doi: 10.1128/JB. 01651-09

Murray, P. R. (1999). Manual of Clinical Microbiology. Washington, DC: ASM Press.

Resch, A., Leicht, S., Saric, M., Pásztor, L., Jakob, A., Götz, F., et al. (2006). Comparative proteome analysis of Staphylococcus aureus biofilm and planktonic cells and correlation with transcriptome profiling. Proteomics 6, 1867-1877. doi: 10.1002/pmic.2005 00531

Resch, A., Rosenstein, R., Nerz, C., and Gotz, F. (2005). Differential gene expression profiling of Staphylococcus aureus cultivated under biofilm and planktonic conditions. Appl. Environ. Microbiol. 71, 2663-2676. doi: 10.1128/AEM.71.5.2663-2676.2005

Rode, T. M., Moretro, T., Langsrud, S., Langsrud, O., Vogt, G., and Holck, A. (2010). Responses of Staphylococcus aureus exposed to $\mathrm{HCl}$ and organic acid stress. Can. J. Microbiol. 56, 777-792. doi: 10.1139/W10-057

Sangari, F. J., Seoane, A., Rodriguez, M. C., Agüero, J., and García Lobo, J. M. (2007). Characterization of the urease operon of Brucella abortus and assessment of its role in virulence of the bacterium. Infect. Immun. 75, 774-780. doi: 10.1128/IAI.01244-06

Schaaff, F., Bierbaum, G., Baumert, N., Bartmann, P., and Sahl, H. G. (2003). Mutations are involved in emergence of aminoglycoside-induced small colony variants of Staphylococcus aureus. Int. J. Med. Microbiol. 293, 427-435. doi: 10.1078/1438-4221-00282

Singh, R., Ray, P., Das, A., and Sharma, M. (2009). Role of persisters and smallcolony variants in antibiotic resistance of planktonic and biofilm-associated Staphylococcus aureus: an in vitro study. J. Med. Microbiol. 58, 1067-1073. doi: 10.1099/jmm.0.009720-0

Young, G. M., Amid, D., and Miller, V. L. (1996). A bifunctional urease enhances survival of pathogenic Yersinia enterocolitica and Morganella morganii at low pH. J. Bacteriol. 178, 6487-6495.

Zhang, Y. (2014). Persisters, persistent infections and the Yin-Yang model. Emerg. Microbes Infect. 3:e3. doi: 10.1038/emi.2014.3

Zhang, Y., Yew, W. W., and Barer, M. R. (2012). Targeting persisters for tuberculosis control. Antimicrob. Agents Chemother. 56, 2223-2230. doi: 10.1128/AAC.06288-11

Conflict of Interest Statement: The authors declare that the research was conducted in the absence of any commercial or financial relationships that could be construed as a potential conflict of interest.

Copyright (C) 2015 Wang, Chen, Chen, Du, Cui, Wu, Zhao, Wu, Zhang, Li and Zhang. This is an open-access article distributed under the terms of the Creative Commons Attribution License (CC BY). The use, distribution or reproduction in other forums is permitted, provided the original author(s) or licensor are credited and that the original publication in this journal is cited, in accordance with accepted academic practice. No use, distribution or reproduction is permitted which does not comply with these terms. 